dere dessen grosse Verdienste um die Flora Spaniens und Deutschlands, um die Forstbotanik und um die Popularisirung der Wissenschaft überhaupt hervorhob. - Ferner besprach derselbe die neueren Arbeiten H. de Vries' und Ludwig's über zwei- und mehrgipfelige Variationscurven, deren Wichtigkeit in entwicklungsgeschichtlicher Hinsicht betonend. - Privatdocent Dr. V. Schiffner hielt einen Vortrag über tropisches Obst und demonstrirte Präparate der wichtigsten in den Tropen als Nahrungsmittel dienenden Früchte.

An dem botanischen Abende am 11. December sprach Prof. Dr. H. Molisch "über die Krystallisation und den Nachweis des Xanthophylls (Carotins) im Blatte" and erläuterte den Vortrag durch prächtige Präparate. - Herr A. Stark referirte über die Untersuchungen Erikson's, betreffend die Beförderung der Keimung der Uredo-Sporen durch niedere Temperaturen. - Prof. Dr. R.v. Wettstein machte einige floristische Mittheilungen und zwar 1. über zwei für Böhmen neue Pflanzen: Prunus fruticans Weihe und Gentiana baltica Murb., deren erstere or bei Karlstein, letztere bei Hohenelbe sammelte; 2. über eine neue europäische Wanderpflanze, Chenopodium carinatum R. Br., die Prof. Makowsky an der Thaya in Mähren auffand und dem Vortragenden zur Bestimmung übersandte; 3. über eine neue Euphrasia, nämlich $E$. Suecica Wettst. et Murb.

In der Monatsversammlung der k. k. zoolog.-botanischen Gesellschaft in Wien, am 8. October, hielt Herr Dr. C. Fritsch einen Nachruf an das verstorbene Mitglied, Stadtgärtner G. Sennbolz; er hob dessen Verdienste um die Horticultur, um die floristische Durchforschung Oesterreichs, dessen warmes Interesse an den Geschicken der Gesellschaft hervor.

\title{
Botanische Sammlungen, Museen, Institute etc.
}

\section{Die Hieracien der Umgebung von Seckau in Ober- Steiermark.}

\section{Hieracia Seckamensia exsiccata}

(Stiria superior).

Von Dr. Gustav v. Pernhoffer (Wien).

II.') (1755).

35. Hieraciam Pilosella Linné. Fl. suec. ed. II, p. 272

Grex XI. Vulgare Näg. et Pet. 1. c. p. 152. - Subspecies angustius? $=H$. Pilosella $\beta$ angustifolium $\mathrm{Tausch}$ in Flora 1828, p. 52. - Conf. Näg. et Pet. l. c. p. 157. - Exs. Nr. 186!

i) Vergl. Jahrg. 1894: S. 315, 362, 430, 477; Nr. 8, 9, 11, 12, 
In declivibus graminosis apricis juxta viam versus sic dict. „Hammerberg" ; c. $870 \mathrm{~m} \mathrm{s.} \mathrm{m.}$

Diese Pflanze, welche mir nur durch das fast zur Regel gehörige Vorhandensein von 2 oder $3(-7)$ Schäften von der Na geli'schen Beschreibung und Exsiccate objger Subspecies abzuweichen scheint, unterscheidet sich durch die Vereinigung folgender Merkmale von allen übrigen um Seckau vorkommenden und zur Ausgabe gelangten Pilosella-Typen:

Schäfte, namentlich die Nebenschäfte, häufig gabelig, meist nur $10-18 \mathrm{~cm}$ hoch und in der Regel t reichlich behaart; Haare ziemlich hell, höchstens an ihrer Basis schwärzlich, 2-3 mm lang; Drüsen an der Schaftspitze zahlreich, sonst aber nur sehr zerstreut und spärlich. Hülle 9-10 mm lang, Schuppen schmal, hell- bis graugrũn, schmalweiss berandet, meist nor armdrüsig und -flockig, aber \pm reichlich behaart; Haare nur gegen die Spitze hell, sonst \pm dunkel bis schwärzlich. Blätter lanzettlich, spitzlich, hellgrün: Blattrücken grünlichgrau oder grau, dichtfilzig; Randblüten röthlich gestreift, Ausläufer verlängert, dicht grauzottig behaart, bäufig mit gabeligen Blütenknospen endigend.

Ich fand diese Pflanze nur neben einem von der bezeichneten Strasse abzweigenden Feldwege unfern der Strassenschenke $u$. $z W$. in Gesellschaft des sub Nr. 65 ausgegebenen H. brachiatum und einiger Exemplare des $\boldsymbol{H}$. Pilosella (3). Dieselbe blühte bereits zu Ende des Monates Juni.

\section{Hieracium Pilosella L. พ. 0.}

Grex XI. Vulgare Näg. et Pet. $=$ H. Pilosella $\alpha$. vulgare Tausch w. 0. - Subspecies?

In graminosis apricis ad marginem silvae quae vulgariter „Kuhbalt" dicitur; c. $860 \mathrm{~m}$ s. m.; solo schistoso.

Ist insbesonders durch die reichliche Flockigkeit der Hülle sowie des - selten gabeligen - Schaftes ausgezeichnet. Schuppen schmal, gewöhnlich hellgrau oder blassgrünlich, schmalweiss gerandet, in der Regel nur sehr mässig drüsig, und so wie der Schaft zerstreut bis mässig behaart. Haare ziemlich hell, an ihrer Basis öfters dunkel bis schwärzlich; selten mit zahlreicheren dunklen Haaren und Drüsen bekleidet und dann die Hülle dunkelgrau bis sehwärzlich. Blätter lanzettlich, spitzlich hellgrün, Blattrücken hellgrau bis fast weisslich filzig. Blüten hellgelb, meist einfärbig oder nur schwachröthlich gestreift. Ausläufer weisslich filzig.

Ganz nahebei, an humösen mit Fichtennadeln bedeckten Waldrändern fand ich gleichzeitig (im Juli) blühend einige wenige Pflanzen, welche sich von den vorigen hauptsächlich durch grössere Köpfchen, mehr breitliche Hüllschuppen, noch stärkere Flockenbekleidung, dicke Ausläufer, schärferes Hervortreten der Nervatur des Blattrückens und 
überhaupt durch einen kräftigeren Habitus unterscheiden und zu Grex IV. Latiusculum N äg. et Pet. l. c. p. 140 gehören dürften. ') 37. Hieracium Pilosella L. w. o. - Grex XI. Vulgare, Näg. et Pet. w. 0. Forma ad Gr. XII. suburinescens, ibid. p. 159 transiens.

In graminosis dumetosis ad marginem silvae "Kuhbalt" dist; c. $840 \mathrm{~m}$ s. m.; solo schistoso.

Gehört zu den bereits in Nr. 5, neben $H$. subvirescens ausgegebenen Uebergangsformen, welche sich gewöhnlich durch den schlanken, öfters über $20(-26)$ cn hohen Schaft, schmal lanzettliche, oberseits dunkelgrüne, zuweilen etwas glaucescirende, unterseits graulichgrüne Blätter, sowie durch verlängerte, dünne und steifliche Ausläufer auszeichnen.

38. Hieraciam Pilosella L. w. o. -- Grex X. Subcaulescens. $\mathrm{N}$ äg. et Pet. l. c. p. 148.

In silvaticis sic dict. "Kuhbalt" (a) et ad pedem montis Kalvarienberg (b); c. $860-880 \mathrm{~m}$ s. m.; solo schistoso.

Die Exemplare a stammen von den gleichen Plätzen wie jene des sub Nr. 4-5 ausgegebenen $H$. Subvirescens; scheinen mir jedoch, nach Vergleichung mit dem von Oborny aus Znaim erhaltenen, $H$. subcaulescens subsp. melanocomum $\mathrm{Näg}$. et $\mathrm{P}$ et. 1. c. p. 150 und mit Rücksicht auf das von Nägeli 1. c. p. 148 hervorgehobene Merkmal des Vorkommens langgestielter Drüsen, eher zu dieser Sippe zu gehören. Noch sicherer dürfte diese Diagnose jedoch auf die, leider nur in sehr geringer Zahl gesammelte Pflanze $b$ passen, deren Hülle durch reichliche und ziemlich lange, sehr dunkle, einfache und Drüsenhaare, welche \pm zerstreut auch den Schaft bekleiden, beinahe sehwarz erscheint. Beide blühen bereits im Monate Juli.

Hieran reihen sich einige, erst im August an grasigen Abhängen aufgefundene Pflanzen zerstreuten Vorkommens, welche eine 10-11 mm lange, 士 obscure Hülle, kaum breitliche, hellrandige Schuppen, stumpfliche oder nur Kurzbespitate Blätter mit höchstens graulichgrüner, sehr dünnfilziger Rückseite, und gewöhnlich einfärbige oder nur schwachröthlich gestreifte Randblüten besitzen; Haare an Schaft und Hülle mässig zahlreich (selten) - 0; Drüsen mitunter ausnahmslos nur kurz gestielt. Von diesen, gleichsam intermediäre Formen der Sippen Subcaulescens? und Vulgare Näg. et Pet. darstellenden Pflanzen ${ }^{2}$ ) zeichnen sich zwei durch ihren gabeligen Schaft aus, und zwar ist derselbe bei einer dieser Pflanzen sowohl in seinem untersten Abschnitte wie auch nahe der Spitze gabelig; Kopfzahl $=4$, bei den anderen nur am Beginne seines obersten Drittheiles; Kopfzahl $=2$. und es endet zugleich ein stengelartiger, verlängerter, bogig aufsteigender Ausläufer mit einem gabeligen 3 köpfigen Knospenstand, wodurch dieses Exemplar bei seiner auch sonstigen Ueppigkeit (Höhe $26 \mathrm{~cm}$ ) dem $H$. brachiatum Nr. 20 einigermassen ähnlich erscheint. 